\title{
Sobrevida de mulheres com câncer de mama, de uma cidade no sul do Brasil
}

\author{
Survival of women with breast cancer, of a city in the south of Brazil
}

Supervivencia de mujeres con cáncer de mama, de una ciudad en el sur de Brasil

\author{
Arlene Laurenti Monterrosa Ayala' \\ IInstituto Superior e Centro Educacional Luterano Bom Jesus. Policlínica do Boa Vista, \\ Secretaria Municipal de Saúde. Joinville-SC, Brasil.
}

Submissão: 19-02-2011 Aprovação: 12-10-2012

\section{RESUMO}

Realizou-se um estudo de coorte retrospectivo para avaliar a sobrevida de 655 mulheres com câncer de mama, atendidas no Sistema Único de Saúde, em Joinville, Santa Catarina, Brasil. Para a análise de sobrevivência aplicou-se o método estatístico de Kaplan-Meier e o modelo de Cox. A sobrevivência das mulheres segundo o estadiamento resultou ser significativamente diferente de acordo com os resultados do Teste Log-rank. A razão de risco instantâneo de morte entre os estádios I/II, I/III e I/IV foi de 3,26, 15,40 e 25,51, respectivamente. Tais achados encontram-se em consonância com os dados da literatura, e apontam que o estadiamento é uma variável que explica as disparidades na sobrevivência entre as mulheres com câncer de mama.

Descritores: Neoplasias da Mama; Estadiamento de Neoplasias; Análise de Sobrevida.

\begin{abstract}
A retrospective cohort study was carried out aiming to evaluate the survival of 655 women with breast cancer, seen in the Single Health System, Joinville, Santa Catarina, Brazil. For the analysis of survival, the method of Kaplan-Meier and Cox model was applied. The survival according to the staging of women was significantly different in accordance with the results of the test of Log-rank Test. The rate of risk of instantaneous death between the stages I/II, I/III and I/IV was 3.26, 15.40 and 25.51, respectively. These findings are in consonance with literature data, and indicate that the staging is a variable that explains the differences in the survival among women with breast cancer.
\end{abstract}

Key words: Breast Neoplasm; Neoplasm Staging; Survival Analysis.

\section{RESUMEN}

Se realizo un estudio retrospectivo de cohortes para avaluar la supervivencia de 655 mujeres con cáncer de la mama, atendidas por el Sistema Único de Salud (SUS), Joinville, Santa Catarina, Brasil. Para el análisis de supervivencia utilizamos el método estadístico de Kaplan-Meier y el modelo de Cox. La supervivencia de las mujeres de acuerdo con la estadificación fué significativamente diferente de acuerdo con los resultados de la Prueba de Log-rank. La tasa de riesgo de muerte instantánea entre las fases I/II, I/III y I/IV fue de 3,26; 15,40 y 25,51, respectivamente. Los resultados encontrados en este estudio están de acuerdo con los datos de la literatura, y indican que la estadificación es una variable que explica las diferencias en la supervivencia entre las mujeres con cáncer de la mama.

Palabras clave: Neoplasias de la Mama; Estadificación de Neoplasias; Análisis de Supervivencia. 


\section{INTRODUÇÃO}

Nos últimos anos a incidência de casos de câncer de mama tem aumentado notadamente no Brasil, e esse crescimento permanece apesar de um maior conhecimento dos fatores de risco, da ampliação dos serviços e de aparatos para diagnóstico dessa enfermidade.

Segundo o Instituto Nacional de Câncer (INCA), o câncer de mama é a segunda causa de morte entre as mulheres brasileiras, e a estimativa de casos novos da doença em nosso país para 2010, é de 49 a cada 100 mil mulheres. Na região sudeste, de acordo com esse Instituto esse tipo de câncer é o mais incidente na população feminina, em torno de 65/100 mil, seguidas das regiões sul (64/100 mil), centro-oeste (38/100 mil) e nordeste $(30 / 100$ mil), sendo que as suas taxas de mortalidade continuam elevadas. Muito provavelmente, pelo expressivo número de diagnósticos realizados em estádios avançados ${ }^{(1)}$.

Autores têm demonstrado que um fator prognóstico importante para a sobrevida das mulheres é o estadiamento no momento do diagnóstico, associando os melhores padrões de sobrevida ao estadiamento inicial ${ }^{(2)}$. Nessa mesma linha, para outros pesquisadores, o diagnóstico do câncer de mama em fases iniciais reduz o risco de morte de forma significativa. Ressaltam ainda que a sobrevida é um parâmetro importante para avaliar resultados na área oncológica, onde as taxas de mortalidade em séries históricas são de alta relevância analítica, sendo possível a análise estatística de sobrevida expressar a probabilidade de vida após um período determinado e revelar a qualidade dos serviços de saúde, como por exemplo, no caso do rastreamento precoce do câncer ${ }^{(3)}$.

A análise de sobrevida, quando desenvolvida com base populacional, contribui para a descrição do comportamento da doença e dos fatores prognósticos a ela relacionados. Segundo os pesquisadores, este tipo de estudo permite ao profissional de saúde e ao paciente conhecer o comportamento da doença, possibilitando uma abordagem realista e que proporcione maior qualidade de vida aos indivíduos acometidos ${ }^{(4)}$.

Considerando que os estudos confirmam a relevância de informações sobre a sobrevida das mulheres com câncer de mama enquanto indicador para servir de apoio, juntamente com outros indicadores na avaliação do impacto dos serviços de saúde, para o diagnóstico precoce e tratamento dessa enfermidade, e por identificar-se a necessidade de produzir indicadores que permitam conhecer a situação do câncer de mama em Joinville-SC, realizou-se por meio deste estudo a análise de sobrevida mediante o exame do indicador de sobrevivência das mulheres com câncer de mama nos estádios I, II, III e IV atendidas pelo Sistema Único de Saúde, no período compreendido entre 2000 e 2009.

\section{MÉTODOS}

Realizou-se um estudo de coorte retrospectivo baseado em uma amostra constituída por mulheres que foram diagnosticadas com câncer de mama, no período compreendido entre 2000 e 2009, em uma Unidade de Especialidades Médicas do Sistema Único de Saúde em Joinville.
A Resolução 196/96 do Conselho Nacional de Saúde (CNS), que trata das Diretrizes e Normas Regulamentadoras de Pesquisas envolvendo Seres Humanos norteou a coleta de dados e o anonimato das mulheres investigadas foi garantido ${ }^{(5)}$.

Os dados coletados foram obtidos nos prontuários e atestados de óbito e foram inseridos em uma planilha Excel, desenhada para o estudo. O número de mulheres investigadas foi de 713; entretanto, foram excluídas 16 devido à ausência de dados no prontuário e 32 por terem sido categorizadas como estádio Tsi (carcinoma in situ). O universo da amostra deste estudo foi composto, portanto, por 655 (IC - 0,99, EA - 0,46) mulheres, em uma proporção estimada da amostra de $70 \%$.

A partir do exame clínico, as mulheres foram estadiadas com base no sistema TNM proposto pela União Internacional Contra o Câncer (UICC) em 1988 ${ }^{(6)}$. Quanto à classificação TNM foi considerado o tamanho do tumor: menor ou igual a $2 \mathrm{~cm}$ (T1), 2 a $5 \mathrm{~cm}$ (T2) e maior que $5 \mathrm{~cm}$ (T3). O comprometimento dos linfonodos: N0 (sem comprometimento dos linfonodos), N1 (metástase em linfonodos axilares homolaterais móveis) e N2 (metástase em linfonodos axilares fixos uns aos outros ou a outras estruturas). E metástase classificada em: M0 (ausência de metástase à distância) e M1 (presença de metástase à distância) no diagnóstico da doença. Para a análise estatística do estadiamento foi utilizado o programa " $R$ " e as variáveis quantitativas foram calculadas por meio de medidas de tendência central e dispersão.

O tempo de sobrevida foi definido como o período entre o diagnóstico de câncer de mama e a ocorrência de óbito. O evento de interesse foi o óbito atribuído apenas ao câncer de mama, segundo a declaração de óbito. Aplicou-se o método estatístico de Kaplan-Meier para obter a probabilidade de sobrevida estimada e a curva de sobrevida acumulada. Para a comparação entre a sobrevivência e distribuição da sobrevivência segundo os estádios foi utilizado o Teste de Log-rank. As probabilidades estimadas de sobrevivência para os diferentes estadiamentos foram traduzidas em anos decimais e sua distribuição foi apresentada por meio de curvas, com intervalo de confiança de 95. Para estimar os efeitos da idade e de cada estadiamento na sobrevida das pacientes utilizou-se o modelo de risco proporcional de Cox, sendo testadas as interações entre as variáveis, I-II, I-III e I-IV e os resultados foram expressos por meio da razão de risco entre elas, levando-se em conta o nível de significância estatística de 0,05.

\section{RESULTADOS E DISCUSSÃO}

As informações da variável idade das mulheres pesquisadas, obtidas pela revisão dos prontuários, demonstraram que a população estudada possuía entre 21 e 90 anos e a média de idade de 55,09 anos $\pm 13,30$. A maior parte das mulheres com câncer de mama encontrava-se na faixa etária de 40 a 50 anos (28\%). Duas séries de casos demonstram esse perfil etário para o câncer de mama, onde a média de idade foi de 56 e 53,85 anos com desvio padrão de 16 e 13,06, respectivamente ${ }^{(7-8)}$.

Com respeito aos estadiamentos, foram encontrados os percentuais de $20 \%$, 48\%, 27\% e 5\% concernentes aos estádios I, II, III e IV. Referente às mulheres investigadas, 32\% possuíam 
a doença localmente avançada ou metastática. Um estudo de base hospitalar com 252 mulheres realizado em Santa Maria/ RS mostrou que $26,6 \%$ da população pesquisada encontravam-se nos estádios clínicos III e IV ${ }^{(9)}$. Outras três pesquisas encontraram percentuais de $13 \%, 23,6 \%$ e $11,2 \%{ }^{(10-12)}$. Ao examinar em conjunto estes percentuais e os de Joinville, constata-se que no município os casos adiantados da doença são elevados, entretanto abaixo, por exemplo, do Estado de Santa Catarina e do Município de Juiz de Fora/MG que apresentam os percentuais de $35,7 \%$ e $34,7 \%$, respectivamente ${ }^{(8,13)}$.

Outro dado igualmente importante identificado em Joinville-SC é que, no primeiro qüinqüênio do estudo (2000-2004), o percentual de casos avançados era de $29,5 \%$, ao passo que, no segundo (2005-2009), este percentual subiu para 33,9\%. Este último dado mostra, no âmbito do setor público de saúde, indícios reveladores da capacidade dos serviços de ampliarem a detecção precoce do câncer de mama. A tendência observada neste estudo ao longo de 10 anos é que o percentual dos casos avançados do câncer de mama vem crescendo no município. Tal tendência contraria os dados de um estudo que trata da evolução temporal dos estádios do câncer de mama em mulheres residentes em Goiânia-GO, no período entre 1989 e 2003, que revela um aumento dos casos de carcinoma in situ e de carcinomas invasores localizados somente na mama em detrimento de uma redução de 17,7\% dos casos avançados no ultimo período investigado ${ }^{(7)}$.

Aqui, é necessário chamar a atenção para algumas pesquisas que tratam do rastreamento populacional para o diagnóstico precoce do câncer de mama - por meio da mamografia - realizado em três países, Estados Unidos, Suíça e Alemanha ${ }^{(14-16)}$. Tais estudos revelam um impacto considerável sobre a diminuição do número de mulheres com diagnóstico de estádio avançado, com conseqüente aumento de sobrevida dessas mulheres.

Tabela 1 - Distribuição do câncer de mama segundo variáveis selecionadas em mulheres no município de Joinville-SC, no período de 2000 a 2009

\begin{tabular}{ccc}
\hline Variáveis & $\mathbf{N}$ & $\%$ \\
\hline Faixa etária & & 1 \\
-30 & 8 & 10 \\
$30 \vdash-40$ & 64 & 28 \\
$40 \vdash 50$ & 182 & 25 \\
$50 \vdash-60$ & 163 & 19 \\
$60 \vdash 70$ & 124 & 17 \\
$70 \vdash$ & $\mathbf{6 5 5}$ & $\mathbf{1 0 0}$ \\
Total &
\end{tabular}

Estadiamento ao diagnóstico

$\begin{array}{ccc}\text { Estadio I } & 131 & 20 \\ \text { Estadio II } & 315 & 48 \\ \text { Estadio III } & 178 & 27 \\ \text { Estadio IV } & 31 & 5 \\ \text { Total } & \mathbf{6 5 5} & \mathbf{1 0 0}\end{array}$

Na tabela 2 constata-se que a sobrevivência das mulheres segundo o estadiamento resultou ser significativamente diferente de acordo com os resultados do teste de log rank, cuja probabilidade (p) esteve abaixo do nível crítico aceitável $=0,05$. O tempo de sobrevida foi superior para o estádio I com uma probabilidade de sobrevida mediana maior do que 120 meses. Para o estádio II o tempo mediano de sobrevida foi de 118 meses. Nos casos dos estádios III e IV a sobrevivência foi menor. O estádio III e IV obtiveram um tempo mediano de sobrevida de $51,26 \pm 2,38$ e 29,05 $\pm 2,23$ meses, respectivamente.

Um conjunto de estudos corrobora os achados desta pesquisa, mostrando que o estadiamento avançado da doença se encontra associado a uma pior sobrevida por câncer de mama $^{(4,7,13,17)}$.

A sobrevida global em Joinville-SC obtida pela estimativa de Kaplan-Meier foi de 57,8\% ao final do período de 10 anos, enquanto que, em cinco anos, a sobrevida estimada foi de $78,6 \%$. A comparação dos percentuais de sobrevida encontrados em outros dois estudos mostra que a sobrevida em 5 anos no município de Joinville-SC é inferior àquela observada no estudo de mulheres com câncer de mama do Hospital Universitário de Santa Maria, no Rio Grande do Sul, que identificou um percentual global de sobrevida de $87,7 \%$. Entretanto, é superior à encontrada numa coorte retrospectiva de base hospitalar com muIheres de New South Wales que identificou 76\%. Com relação a 10 anos, ambas pesquisas revelam percentuais de sobrevida acima de Joinville-SC, àquele mostra o percentual de 78,7\%, ao passo que esta exibe em seus achados o percentual de $65 \%(9,18)$.

Estratificando-se as pacientes de acordo com os estádios constatamos diferenças significativas de sobrevivência entre elas de acordo com o valor estatístico do Teste de Log-rank que foi de 7,81, com grau de liberdade três e valor de p associado de 0,0001. A probabilidade de sobrevida para os estádios I, II, III e IV foi de $94 \%, 66 \%, 41 \%$ e $0 \%$, respectivamente, ao final do período de 10 anos, enquanto que em cinco os percentuais apontam para $97 \%, 88 \%, 51 \%$ e $17 \%$. Constata-se uma queda acentuada das probabilidades de sobrevida do estádio III e IV quando comparadas aos estádios I e II.

Três estudos que apresentam a sobrevida das mulheres com câncer de mama segundo os estádios permitem a confrontação com a situação encontrada em Joinville. O primeiro, realizado no Hospital Universitário de Santa Maria, no Rio Grande do Sul, mostra os seguintes percentuais de sobrevida em cinco anos para as pacientes nos estádios I, II, III, e IV: 97\%, 93\%, $73 \%$ e $57 \%$, respectivamente ${ }^{(9)}$. O segundo, que trata de uma coorte de base hospitalar com pacientes do sexo feminino, em Juiz de Fora-MG, indica as porcentagens de 92,7\%, 88,3\%, $67 \%$, 54\% para os estádios acima referidos ${ }^{(13)}$. A comparação dos percentuais dos estádios II, III e IV desses dois estudos mostra que estes são superiores aos percentuais encontrados em Joinville-SC. Por último, comparando-se com um terceiro estudo, realizado na Irlanda com os percentuais de $91 \%$, 83\%, $72 \%$ e $11 \%{ }^{(17)}$, constata-se que as mulheres em Joinville-SC, tiveram uma sobrevida superior àquelas nos estádios I, II e IV. Entretanto, para as mulheres no estádio III a sobrevida foi inferior.

Em relação ao modelo de riscos proporcionais de Cox para a variável estádio, observou-se que a hazard risco instantâneo 
Tabela 2 - Sobrevida das mulheres com câncer de mama segundo estadiamento patológico I, II, III e IV. Joinville, 2000 a 2009.

\begin{tabular}{|c|c|c|c|c|c|c|c|}
\hline \multirow{2}{*}{$\begin{array}{l}\text { Estádio } \\
\text { patologico }\end{array}$} & \multirow{2}{*}{$\begin{array}{l}\text { Período } \\
\text { em anos }\end{array}$} & \multirow{2}{*}{ Em risco } & \multirow{2}{*}{ Censura } & \multirow{2}{*}{ Evento } & \multirow{2}{*}{$\begin{array}{l}\text { Sobrevida } \\
\text { estimada }\end{array}$} & \multicolumn{2}{|c|}{0,95 de intervalo de confiança } \\
\hline & & & & & & \multicolumn{2}{|c|}{ Lim. mín. Lim. máx. } \\
\hline \multirow{5}{*}{$\begin{array}{c}\text { Estadio } \\
\quad \text { I }\end{array}$} & -1 & 131 & 31 & 0 & 1 & 0,9644 & 1 \\
\hline & $1 \vdash 4$ & 100 & 32 & 1 & 0,99 & 0,9482 & 0,9990 \\
\hline & 4 ト 7 & 67 & 47 & 3 & 0,9456 & 0,8878 & 0,9758 \\
\hline & 가 9 & 17 & 12 & 0 & 0,9456 & 0,8878 & 0,9758 \\
\hline & $9 \mathrm{H} 10$ & 5 & 5 & 0 & 0,9456 & 0,8878 & 0,9758 \\
\hline \multirow{5}{*}{$\begin{array}{l}\text { Estádio } \\
\quad \text { II }\end{array}$} & -1 & 315 & 30 & 3 & 0,9904 & 0,9700 & 0,9975 \\
\hline & 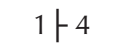 & 282 & 89 & 24 & 0,9061 & 0,8671 & 0,9349 \\
\hline & $4 \vdash 7$ & 169 & 104 & 11 & 0,8471 & 0,8015 & 0,8841 \\
\hline & 가 9 & 54 & 39 & 6 & 0,7530 & 0,7009 & 0,7989 \\
\hline & $9 \mathrm{H} 10$ & 9 & 8 & 1 & 0,6693 & 0,6140 & 0,7205 \\
\hline \multirow{5}{*}{$\begin{array}{c}\text { Estádio } \\
\qquad \text { III }\end{array}$} & -1 & 178 & 23 & 18 & 0,8988 & 0,8425 & 0,9372 \\
\hline & 1 - 4 & 137 & 39 & 45 & 0,6036 & 0,5274 & 0.6752 \\
\hline & $4 \vdash 7$ & 52 & 30 & 13 & 0,4555 & 0,3814 & 0.5316 \\
\hline & 가 9 & 9 & 6 & 1 & 0,4100 & 0,3377 & 0,4862 \\
\hline & $9 \mathrm{H} 10$ & 2 & 2 & 0 & 0,4100 & 0,3377 & 0,4862 \\
\hline \multirow{4}{*}{$\begin{array}{l}\text { Estádio } \\
\text { IV }\end{array}$} & -1 & 31 & 2 & 6 & 0,8064 & 0,6194 & 0,9187 \\
\hline & 1 - 4 & 23 & 4 & 13 & 0,3506 & 0,1950 & 0,5421 \\
\hline & $4+7$ & 6 & 0 & 3 & 0,1753 & 0,0696 & 0,3604 \\
\hline & $7 \mathrm{H} 9$ & 3 & 3 & 0 & 0,1753 & 0,0696 & 0,3604 \\
\hline
\end{tabular}

Estádio I/II Logrank - 6,292 gl - $3 \mathrm{p}=0,012$

Estádio I/III Logrank - 34,992 gl - $3 \mathrm{p}=0,0001$

Estádio I/IV Logrank - 42,579 gl - $3 \mathrm{p}=0,0001$

de morte das mulheres em Joinville nos estádios II, III e IV em relação ao estádio I foi maior em 3,26 (IC95\%: 1,29-8,22; Teste Log-rank, $\mathrm{p}=0$,012) para o II; 15,40 (IC95\%: 6,22-38,12; Teste Log-rank, $\mathrm{p}=0,0001)$ para o estádio III; e 25,51 (IC95\%: 9,64-67,51; Teste Log-rank, $p=0,0001$ ) para o estádio IV.

Uma série de casos que investiga a proporcionalidade do risco de morte por câncer de mama segundo o estádio revela menores índices de risco em relação aos encontrados em Joinville. Essa série mostra que o risco de óbito das mulheres em estádio III é 7,18 vezes maior, e as em estádio IV o risco é de 19,49 vezes maior que as em estádio I ${ }^{(8)}$. Ao comparar os resultados de Joinville com essa série observa-se que as mulheres no estádio III tiveram 8,22 e as em estádio IV, 6,02 vezes menos risco de morte em relação às mulheres de Joinville-SC

Em se tratando da variável idade, o modelo de risco proporcional de Cox demonstrou que não houve significância estatística na curva de sobrevida para as diferentes idades consideradas, dado que o valor estatístico do Teste Log-rank foi de 0,58 e o valor de $\mathrm{p}$ associado foi de 0,4461 . Corroborando, desta forma, uma coorte histórica de 10 anos de seguimento de mulheres com câncer de mama pelo serviço de oncologia do Hospital Geral da Cidade do México, que indica que a idade não é um fator prognóstico significativo para a determinação da sobrevida $^{(19)}$. Entretanto, discorda de outros estudos que apontam a influência da idade para o prognóstico de sobrevida. Segundo estas investigações, as mulheres com 40 a 50 anos apresentam melhor prognóstico quando comparadas às mais jovens ${ }^{(8,20)}$.

\section{CONCLUSÕES}

Os achados deste estudo encontram-se em consonância com os dados da literatura, apontando que o estadiamento do câncer de mama é uma variável importante que explica as disparidades na sobrevivência entre as mulheres portadoras desse agravo.

Os resultados mostraram, no âmbito do setor de Saúde em Joinville-SC, a problemática condição de saúde das mulheres com câncer de mama no município. A expressiva incidência de casos avançados reforça a necessidade de implantar medidas para detecção do câncer de mama em estádios os mais precoces possíveis, como forma de diminuir a mortalidade e aumentar a qualidade de vida das mulheres acometidas por esta doença.

Por fim, por se tratar de um problema que em Joinville-SC tem uma magnitude importante, seria notável e desejável que fossem realizados estudos que aprofundassem a análise com maior precisão de outros fatores que poderiam estar contribuindo para a diminuição importante de sobrevida observada neste estudo. As possibilidades de análise estão, portanto, totalmente abertas. 


\section{REFERÊNCIAS}

1. Instituto Nacional de Câncer. Sismama. Informação para o avanço das ações de controle de câncer de mama no Brasil. [monografia na internet]. 2010 [acesso em $10 \mathrm{dez}$ 2010] Disponível em: < http://www.inca.gov.br/conteudo_view.asp?id = 1932>

2. Abreu E, Koifman S. Fatores prognósticos no câncer da mama feminino. Rev Bras Cancerol 2002;48(1): 113-31.

3. Pedersen L, Gunnarsdottir KA, Rasmussen BB, Moeller $\mathrm{S}$, Lanng $\mathrm{C}$. The prognostic influence of multifocality in breast cancer patients. Breast 2004;13(3):188-93.

4. Bustamante MT, Faerstein E, Latorre MR. Técnicas de análise de sobrevida. Cad Saúde Pública 2002; 18(3):579-94.

5. Conselho Nacional de Saúde (Brasil). Resolução n. ${ }^{\circ} 196$ de 10 de outubro de 1996. Diretrizes e Normas Regulamentadoras de Pesquisas Envolvendo Seres Humanos. Diário Oficial da União 10 out 1996.

6. International Union Against Cancer. TNM classification of malignant tumours. A John Wiley \& Sons, INC Publication; 2002. p. 227.

7. Martins E, Freitas-Junior R, Curado MP, Freitas NMA, Oliveira JC, Silva CMB. Evolução temporal dos estádios do câncer de mama ao diagnóstico em um registro de base populacional no Brasil central. Rev Bras Ginecol Obstet 2009;31(5):219-23.

8. Schneider IJC, Dorsi E. Sobrevida em cinco anos e fatores prognósticos em mulheres com câncer de mama em Santa Catarina, Brasil. Cad Saúde Pública 2009;25(6):1285-96.

9. Moraes AB, Zanini RR, Turchiello MS, Riboldi J, Medeiros LR. Estudo da sobrevida de pacientes com câncer de mama atendidas no hospital da Universidade Federal de Santa Maria, Rio Grande do Sul, Brasil. Cad Saúde Pública 2006;22(10):2219-28.

10. Yu, Xy. Socioeconomic disparities in breast cancer survival: relation to stage at diagnosis, treatment and race. BMC Cancer [periódico na internet]. 2009 out [acesso em 10 dez 2010];18(10):2579-99 Disponível em: <http://www. biomedcentral.com/1471-2407/9/364 $\geq$

11. Macchetti AH. Estadiamento do câncer de mama diagnosticado no Sistema Público de Saúde de São Carlos. Medicina (Ribeirão Preto) 2007;40(3):394-02.

12. Keating NL, Landrum MB, Ayanian JZ., Winer EP, Guadagnoli E. The Association of Ambulatory Care with Breast
Cancer Stage at Diagnosis Among Medicare Beneficiaries. J Gen Intern Med [periódico na internet]. 2005 Jan [acesso em 10 dez 2010];20(1): 38-44 Disponível em: $<10.1111 /$ j.1525-1497.2004.40079.x>

13. Guerra MR, Mendonça GAS, Teixeira MTB, Cintra JRD, Carvalho LM, Magalhães LMPV. Sobrevida de cinco anos e fatores prognósticos em coorte de pacientes com câncer de mama assistidas em Juiz de Fora, Minas Gerais, Brasil. Cad Saúde Pública 2009;25(11):2455-66.

14. Nass SJ, Henderson C, Lashof JC. Mammography and Beyond: developing technologies for the early detection of breast cancer [monografia na internet]. Washington: National Research Council; 2001. [acesso em 10 dez 2010] Disponível em: <www.nap.edu/books/0309072832/ $\mathrm{html}>$

15. Bulliard JL, Ducros C, Jemelin C, Arzel B, Fioretta G, Levi F. Effectiveness of organised versus opportunistic mammography screening. Ann Oncol [periódico na internet]. 2009 Jul [acesso em 10 dez 2010];20(7):1199-02 Disponível em: <10.1093/annonc/mdn770>

16. Katalinic A, Bartel C, Raspe H, Schreer I. Beyond mammography screening: quality assurance in breast cancer diagnosis. Br J Cancer [periódico na internet]. 2007 Jan [acesso em 10 dez 2010];15;96(1):157-61 Disponível em: $<$ www.bjcancer.com $\geq$

17. Blacam C, Gray J, Boyle T, Kennedy MJ, Hollywood D, Butt J, et al. Breast cancer outcomes following a national initiative in Ireland to restructure delivery of services for symptomatic disease. Breast 2008; 17(4):412-7.

18. Taylor R, Davis P, Boyages J. Long-term survival of women with breast cancer in New South Wales. Eur J Cancer 2003;39(2):215-22.

19. Luna LL, Salazar EM, Torres RMD, Mejía GT, Ruiz PA, Ponce EL. Factores pronósticos relacionados con la supervivencia del cáncer de mama. Salud Pública Méx [periódico na internet]. 2008 Abr [acesso em $10 \mathrm{dez}$ 2010];50(2):119-25 Disponível em: <http://www.scielo.org.mx/scielo.php?script =sci_arttext\&pid =S0036$-36342008000200005 \& \operatorname{lng}=\mathrm{es}>$

20. Santi M, Capocaccia R, Verdecchia A, Esteve J, Gatta G, Micheli A, et al. Survival of women with breast cancer in Europe: variation withage, year of diagnosis and country. Int J Câncer 1998; 77:679-83. 